\title{
Long distance travel for routine elective surgery: questionnaire survey of patients' attitudes
}

\author{
G P Howell, D Richardson, A Forester, J Sibson, J M Ryan, B T Morgans
}

\begin{abstract}
Objective-To carry out an-audit and assess the acceptability of long distance travel to patients on a lengthy surgical waiting list.

Design-Audit and questionnaire survey.

Setting-Military hospital in Wiltshire.

Patients-116 Patients on a general surgical waiting list at Leighton Hospital near Crewe.

Interventions-Preselection for medical fitness at a waiting list review clinic.

Results-Roughly half of patients offered the facility of travelling 120 miles $(190 \mathrm{~km})$ for their routine elective operation at the health authority's expense accepted. The average waiting list time declared by the patients who travelled was 28 months, and 13 patients stated that their condition prevented them from working. Only four patients regretted their decision to participate in the scheme. Lack of visitors did not cause undue concern.

Conclusions-Some patients did not agree to travel for their operation but almost all of those who did so found the scheme satisfactory. Some minor problems were encountered but these could probably be overcome by ensuring use of appropriate transport, extending the postoperative stay when necessary, and more careful selection of patients for an anaesthetic. For efficient use of theatre time in such schemes it is crucial that the operating consultant surgeon should be in charge of case selection.
\end{abstract}

\section{Introduction}

Princess Alexandra Hospital, jointly staffed by Royal Air Force and army medical personnel, treats military and NHS patients in roughly equal numbers. An internal audit of the general surgical unit's work for the first six months of 1989 showed that of 1250 patients having operations, $559(45 \%)$ were military personnel and $691(55 \%)$ NHS patients referred principally from the local area. Families of service personnel make up less than $10 \%$ of the NHS total.

The treatment of NHS patients at the hospital attracts no remuneration from local health authorities. In 1988, however, in line with advice from the Commons Public Accounts Committee,' an initiative was arranged between the hospital (on behalf of the Ministry of Defence) and the Crewe Health Authority to admit patients awaiting routine elective surgery at Leighton Hospital. In the Crewe area health budgets have consistently been underfunded in relation to RAWP (Resource Allocation Working Party) guidelines, contributing to long surgical waiting lists.

Princess Alexandra Hospital is used to receiving patients from a distance. The catchment area for military personnel in the United Kingdom is extensive, and patients are regularly admitted from around the world via Royal Air Force aeromedical flights.

\section{Subjects and methods}

Patients from the Crewe area on a general surgical waiting list at Leighton Hospital were first reassessed at a review clinic by their local consultant surgeon. Those who were found still to require surgery and in whom a history and brief anaesthetic assessment showed that they were fit for operation were then offered two options: (a) to return to the waiting list with an intention of proceeding to surgery within one year or $(b)$ to travel at the health authority's expense to have their operation at Wroughton. About half of those thought suitable agreed to travel.

In the five months between November 1988 and April 1989, 116 patients travelled the 120 miles $(190 \mathrm{~km})$ from Crewe to the Princess Alexandra Hospital at RAF Wroughton for their operation. Transport (minibus or car) collected the patients from home on Sundays and Thursdays and returned from Wroughton the same day with patients who had had their operations after previous transfers. Patients awaited the next shuttle if they had not fully recovered from their operation.

Patients who returned home with sutures had these removed at the general practitioner's surgery, and surgical outpatient review was carried out by the surgeon at Leighton Hospital. Patients were told to refer any postoperative problems to their general practitioner. Patients took home with them a handwritten discharge letter, and a typed summary followed in due course.

At the conclusion of the scheme a questionnaire, letter of explanation, and a stamped addressed envelope were sent to each patient. The questionnaire consisted of 26 questions to which patients were asked to respond by deleting options and adding script if they wished. One reminder letter was sent to those who did not reply within six weeks.

\section{Results}

On arrival at the Princess Alexandra Hospital four of the 116 patients were found to be unfit for an anaesthetic and were returned home. Two of these patients had pronounced glycosuria, one had unacceptable hypertension, and one had an exacerbation of chronic obstructive airways disease. The remaining 112 patients were subjected to 114 procedures (table I)

Forty three of these were single or bilateral inguinal hernia repairs, and most of the remainder (61) were varicose vein operations. Thirty three of these were bilateral Trendelenburg procedures with local ties, avulsions, or long saphenous vein strips. In 47 of the 112 operations the principal surgeon was a consultant, in 31 the senior registrar, and in 34 a registrar.

One hundred and thirteen of the 116 questionnaires

TABLE I - Operations performed $($ total $=114(112$ patients $))$

\begin{tabular}{|c|c|c|c|}
\hline & No & & No \\
\hline Unilateral inguinal hernia & 34 & Gynaecomastia excision & 1 \\
\hline Bilateral inguinal hernia & 9 & $\begin{array}{l}\text { Hydrocele } \\
\end{array}$ & 1 \\
\hline Unilateral varicose veins & 28 & Epididymal cyst excision & 1 \\
\hline Bilateral varicose veins & 33 & Multiple sebaceous cysts & \\
\hline Cholecystectomy & 1 & of scalp & 1 \\
\hline Submandibular stone & & Haemorrhoidectomy & i \\
\hline extraction & 1 & Sigmoidoscopy and anal & \\
\hline Epigastric hernia & 1 & stretch & 1 \\
\hline Circumcision & 1 & & \\
\hline
\end{tabular}

Howell, Department of Infirmary, Glasgow G4 OSF.

Br.Med F 1990;300:1171-3 
were returned (response rate $97 \cdot 4 \%$ ). Two of the questionnaires were sent in by patients who were unfit for operation and who returned home on the day of arrival; they were therefore unable to respond to some of the questions. No questionnaires were otherwise returned incomplete or spoilt.

Waiting list-Patients were asked how long they had been waiting for their operation. The average time was 28 months and the longest 96 months. The mean waiting time for inguinal hernia repair was 32 months and for varicose vein operations 25 months. Seventy of the 113 patients regarded their operations as routine and 34 as urgent. These included operations for hernias and varicose veins in almost equal numbers. The remainder thought that their operations (for varicose veins (eight cases) and excision for gynaecomastia (one)) were cosmetic. Thirteen patients reported that they were unable to work because of their symptoms. Of these patients, seven had inguinal hernias and five varicose veins; the remaining patient had a phimosis.

Travel arrangements and duration of hospital staySeventy six patients enjoyed the journey from Crewe. Thirty seven found it tolerable, and no patient found it intolerable. One hundred patients travelled by minibus and 13 by car. The duration of the journey from home to hospital, which included an initial visit to five homes, varied from three to nine hours. On the longer journeys the driver got lost. The average journey time was 3 hours 50 minutes. Patients returned home two or three days after operation, the longest stay being eight days. Though assessed by a surgeon as recovered enough to travel, 16 of 111 patients considered that they were unfit to return home when they did, and in general the trip back was less well tolerated. Fifty two of the 111 found the return journey comfortable and 38 tolerable, but 20 were uncomfortable and one found the journey agonising. Most patients were given oral analgesia for the return journey, but 18 of the 59 who were uncomfortable were not. Eighty nine of the 111 patients travelled home by minibus and the remainder by car. Travellers in cars suffered greater discomfort.

Response to visitors - Ninety one patients received no visitors during their stay. Of these, 73 were not at all concerned, 15 were mildly concerned, and only three were very concerned. When asked how they would tolerate specified periods in hospital without visitors 85 of 111 patients said that they would tolerate seven days, 46 that they would tolerate 14 days, and 36 that they would tolerate 21 days.

Postoperative progress-Twenty seven patients identified a problem after their return home which they attributed to their operation. Twenty one called their doctor, three saw the district nurse, and three did not seek advice. Only one patient, however, was referred for an earlier hospital review. Patients having inguinal herniorrhaphy reported a high problem rate, 11 of 41 developing a "complication" as perceived by the patient. Six patients with hernia developed inflamed wounds, some of which were treated with antibiotics by the general practitioner and some required dressings. Two patients with hernia developed pronounced bruising associated with later serous discharge reflecting haematoma formation, and three patients developed tender swelling of a testicle; this was particularly troublesome in one patient, necessitating earlier surgical outpatient review. Sixteen of 61 patients having varicose vein surgery reported problems once they had returned home. Ten patients developed inflamed wounds, some of which were treated with antibiotics by the general practitioner and some required dressings. Six patients with varicose veins complained of leg pain, the cause of which was not clear.
Patients' rating of scheme-Patients were asked whether they would travel to Wroughton for a further operation in similar circumstances. Only four patients said that they would not. Three of these patients had developed a complication and had a less than optimum result after varicose vein surgery, and one had a haematoma after an inguinal hernia repair. All, however, said that they would recommend the scheme to a friend or relative.

Waiting times for operation that patients would tolerate before preferring to travel-When asked how long they would wait for a routine operation locally before opting to travel to another location 44 of 113 patients said that they would rather travel than wait more than one month, 60 that they would rather travel than wait more

TABLE II-Waiting list times that patients would tolerate before preferring to travel for operation (113 patients)

\begin{tabular}{lcccccccc}
\hline & \multicolumn{1}{c}{ Waiting list time (months) } \\
\cline { 2 - 9 } & 1 & 2 & 3 & 6 & 9 & 12 & 18 & 24 \\
\hline No preferring to travel & 44 & 49 & 60 & 84 & 92 & 103 & 104 & 113
\end{tabular}

TABLE III-Distances that patients would be willing to travel for operation (113 patients)

\begin{tabular}{lcccc}
\hline & \multicolumn{4}{c}{ Miles $(\mathrm{km})$} \\
\cline { 2 - 5 } & $50(80)$ & $100(160)$ & $200(320)$ & $300(480)$ \\
\hline No willing to travel & 102 & 88 & 75 & 75. \\
\hline
\end{tabular}

than three months, 103 that they would rather travel than wait more than 12 months, and all 113 that they would rather travel than wait two years (table II). Distance was not a problem, 102 of 113 patients indicating a willingness to travel 50 miles $(80 \mathrm{~km})$ and 75 of the 113 a willingness to travel 300 miles $(480 \mathrm{~km})$ (table III).

\section{Discussion}

Many assumptions have been made about patients' attitudes to travelling for routine elective surgery but there is little researched material. This survey clearly does not represent a random cross section of the community but rather a group of patients who had already volunteered to travel for and had undergone an operation. Patients who were very much in favour of local treatment and who declined to travel were not assessed.

The response to our questionnaire shows that a large number of patients would rather travel for an operation than wait to have it performed locally. Of our selected group of 113 patients, 60 would opt to travel for a routine operation rather than wait more than three months for local treatment. Seventy five of the 113 would be happy to travel up to 300 miles $(480 \mathrm{~km})$, and 85 of 111 patients said that they would tolerate seven days in hospital without visitors. These findings concur with a survey by the Northern Regional Health Authority and Gateshead Health Authority of patients from the Harrogate area travelling 50 miles $(80 \mathrm{~km})$ to the Duchess of Kent's Military Hospital at Catterick for elective routine surgery in 1987-8.

Travel arrangements were generally satisfactory, though the return journey was unpleasant for many patients. Possibly the length of inpatient stay may have to be extended for patients in travelling schemes. Over half of the patients found the return trip less than comfortable, and $16(14 \%)$ considered that they were unfit to travel when they did. Transport with adequate leg room is advantageous, particularly after varicose 
vein surgery, and a minibus was preferred. Analgesia should be provided in all cases.

The high incidence of patients seeking advice from the general practitioner or district nurse after their return home was worrying and is not a problem of which we are aware in our normal practice. Complications perceived by patients may not necessarily be regarded as complications by a surgeon but, nevertheless, we were unhappy to discover potential infection rates of around 15\% after inguinal hernia and varicose vein surgery. Patients having this type of surgery remain in hospital for only a day or so. Inflammatory and infective sequelae are likely to have resolved and may be forgotten by the patient by the time of the surgical outpatient review at two or three months. We intend to survey our patients treated locally to see if the trend can be confirmed.

Transient testicular swelling in three patients after inguinal hernia repair was attributable to one surgeon whose practice was probably to overtighten the internal inguinal ring. Personal technique has been modified.

An unexpected finding highlighting the cost of waiting lists was that 13 patients $(11 \cdot 5 \%)$ awaiting routine elective surgery claimed that they were unable to work.

Though patients rated the scheme highly, the scheme was not without difficulties. Nevertheless, the apparent success of the scheme challenges the traditional approach of initial assessment, operation, and follow up being performed by the same surgical team. Problems might be expected in matters of contentious management and certainly some patients had slightly different operations from those recommended by the referring surgeon. The preoperative ward round must therefore be conducted with the importance and the duration of the outpatient consultation.

When complications occur they are best dealt with by the operating surgeon and may not be appreciated when review is carried out elsewhere.

Patients were generally allocated for transfer to Wroughton by availability and geographical clustering rather than by the nature of the operation and expected duration of the procedure. Problems were therefore encountered with the content and duration of some operating lists. It is crucial for the efficient use of theatre time and the appropriate allocation of surgical staff that case selection should be made by the operating consultant surgeon and not by administrative personnel.

Selection of patients with regard to their fitness for an anaesthetic before transfer is important to spare patients disappointment and a wasted journey. Four patients in our series were returned home without an operation despite prior assessment at the review clinic. An ideal scheme should include advice from the anaesthetic department of the receiving hospital of local criteria and thresholds for deferring operation in the presence of conditions such as hypertension or glycosuria.

With regard to overall surgical performance it is not possible to get something for nothing. The rate limiting step in surgical performance in this military hospital is operating time rather than bed space, and as a result of operating on 112 patients from another region the same number of local NHS patients in Wiltshire were deferred.

From the operating team's point of view there was no training benefit. The type of routine, repetitive surgery transferred was that which will inevitably be found at low priority on all surgical waiting lists and which we see regularly from day to day. The hospital gained because the Crewe Health Authority contributed $£ 36$ per patient per day based on recovery of minimal costs. This amount might seem to undersell the services offered but compared favourably with the existing arrangements of non-sponsorship of local NHS patients. If similar financial arrangements were to be negotiated with health authorities in the local area then there would be no reason to receive elective surgical patients from far away. 1 Warden J. Service hospitals. Br.Med $1989 ; 299: 10-1$.
2 Johnson M, Donaldson LJ. Northern Regional Health Authority and
Gateshead Health Authority report: national waiting list initiative with the
Duchess of Kent's Military Hospital, Catterick. 1988. (Circulated to all
military hospitals by assistant surgeon general (service hospitals).)

\section{Trends in admissions for hip fracture in England and Wales, 1968-85}

\section{T D Spector, C Cooper, A Fenton Lewis}

Department of Preventive Medicine, St Bartholomew's Hospital Medical College, London EC1M 6BQ

T D Spector, MD, lecturer

Rheumatology Unit, Bristol Royal Infirmary, Bristol

C Cooper, $\mathrm{MD}$, senior registrar

Department of Health,

London

A Fenton Lewis, PHD, senior medical officer

Correspondence to:

Dr Spector.

Br.Med f 1990:300:1173-4
Evidence from several countries indicates that the age specific incidence of hip fracture has increased over the past three decades.' This and the increasing numbers of elderly people have led to predictions of a doubling of the number of hip fractures before the end of the century in most countries. Previous reports from the United Kingdom have confirmed these trends from the mid-1950s to the mid-1970s. ${ }^{2}$ We analysed data on fractured neck of femur obtained in the Hospital InPatient Enquiry for England and Wales from 1968 until 1985 (the last year of the inquiry)

\section{Methods and results}

We obtained data on 64511 patients who died in or were discharged from hospital after sustaining a fractured neck of femur (ICD 820) during 1968-85 and used them as a proxy for admissions, assuming that they were a consistent sample of Hospital Activity
Analysis data. Analysis started at 1968 to avoid early problems of the Hospital In-Patient Enquiry. Data for Wales were not collected after 1982, and we therefore estimated rates accordingly. We calculated standardised admission ratios for men and women aged over 44 , using the age specific rates in 1968 as the reference $(=100)$. The rates increased steadily in women by $61 \%$ up to 1978 and in men by $73 \%$ until 1979 (figure). No further increases occurred in either sex, suggesting that the rates levelled off. The ratio of women to men with hip fracture remained stable at about $4: 1$ over the period. Correspondingly, the rates for men varied more from year to year owing to the smaller numbers of cases.

\section{Comment}

These data suggest that the dramatic increase in the number of hip fractures over recent decades has begun to tail off. The accuracy of data obtained in the Hospital In-Patient Enquiry as an estimate of incidence and regional variations in incidence has been questioned, though any biases probably operated continuously over the 17 years for the whole country. If the data are accepted as reliable they seem to confirm earlier reports of an increase in cases in the United Kingdom since 1958. ${ }^{3}$ The reasons for this increase are, however, unclear, and many hypotheses have 